\title{
Wireless Network Smart Grids for Continuous Power Supply and Monitoring
}

\author{
Suresha, Parvathi C, Ashritha C, Abhay R Muni
}

\begin{abstract}
Advent of electric power revolutionized the life of a civilized human. From the early dated discoveries of Nikola Tesla, Alessandro Volta to the latest applications of electricity has made it indispensable for our daily life. The wide-ranging applications of power have made ease of living. From greenland to brownland we are dealt with the utilization of the electricity. Power generation, transmission, distribution, and efficient utilization promote the progress of a nation. Power crisis which has been abating. India suffers large deficits which need to be overcome at the earliest. We see a lot of power outages and most of the time we never know the reason behind it. Even in the 21st century, the digital age, we should not be facing this problem. One of the reasons behind the shortfall of a proper power supply is the inefficient power monitoring ensued by breakdowns, repairs and maintenance work of power plants, transformers, and transmission lines. This project is designed as the one-time solution to monitor the outages in real time without the requirement of a meatware. A power monitoring system automatically analyzes and retrieves the power quality events. The monitoring system comprises of smart grids and a centralized workstation. Smart grids contribute towards sending data of power supply status to the workstation continuously. Based on the status drawn by the grids the workstations analyze the data and intimate the electricity boards about the power outages. Thus elimination of manual summoning the designated authorities by automatic monitoring acts as the core of the proposed project. The automatic switching to an alternative resource is done by the automatic phase selector. The smart grids also promote to increase efficiency and energy conservation. The gravity of the paper is power monitoring and shift to a secondary power resource in the absence of the primary. Power monitoring acts as a preventive measure to recover the huge deficits suffered by the power crisis.
\end{abstract}

Keywords: Blackouts, Centralized workstation, Global System for Mobile communications(GSM), Power Crisis, Smart Grids, Step Down Transformer(SDT), Wireless Networks (WN)

Manuscript published on 30 September 2019

* Correspondence Author

Dr. Suresha*, Principal, Department of Computer Science and Engineering, Sri Venkateshwara College of Engineering, Bangalore, India. Email: principal@svcengg.edu.in

Parvathi C, Department of Computer Science and Engineering, Sri Venkateshwara College of Engineering, Bangalore, India.

Email: pvc.svce@gmail.com

Ashritha C, Department of Computer Science and Engineering, Sri Venkateshwara College of Engineering, Bangalore, India. Email: ashrithamanjula@gmail.com

Abhay R Muni, Department of Computer Science and Engineering, Sri Venkateshwara College of Engineering, Bangalore, India. Email: abhayrudramuni@gmail.com

(c) The Authors. Published by Blue Eyes Intelligence Engineering and Sciences Publication (BEIESP). This is an open access article under the CC-BY-NC-ND license (http://creativecommons.org/licenses/by-nc$\underline{\mathrm{nd} / 4.0 /)}$

\section{INTRODUCTION}

Electricity is one of the prominent and essential commodities that act as the forte of every industrialized society and economy of a nation. Blackouts are common or even normal routine for most of the developing and underdeveloped countries where electric power generation is underfunded and its infrastructure is poorly managed. The increased population has increased the dependency on continuous power supply in various domains like manufacturing, industrial production, electronics and mundane tasks of mankind. India is also one such nation which is facing a severe power crisis. The power generated from various sources like water turbines, windmills are drawn through transmission lines for domestic usage. These transmission lines play a vital role in extending electric power from the power stations to the doorstep. The transmission lines taken from transformers are severely exposed to the varied conditions of weather like high temperature, wind, heavy rains, thunder-storms, humidity and other natural forces. These have become a threat to the safe operation of transmission lines. In addition to these, pollution due to industrialization, urbanization, scientific method of agriculture is hampering the normal functioning of transmission lines. These transmission lines are critical as they carry high voltages, therefore their operating environment and states are highly complex which require automatic monitoring, a more controlled and protective atmosphere to instantly send alarms when accidents occur and dispatch strategy adjustment according to the operation mode. Thus, the faults will be eliminated or isolated in the small range at the early phase. Primitive transmission lines do not meet the communication needs of the online monitoring of transmission lines. Wireless networks (WNs) have the ability to adapt to severe climatic conditions, cover a large area and are self-organized and self-configured. Proper intelligence and investment in smart grids paves the way in monitoring the power quality events and also achieve synchronization of various resources instantly [1], [4].

\section{RELATED WORK}

In October 1991, the national grids[2] were introduced in order to connect the power stations and major substations from all over the nation to ensure that electricity generated anywhere in mainland India can be used to satisfy demand elsewhere. 


\section{Wireless Network Smart Grids for Continuous Power Supply and Monitoring}

This has been a major move by the state-owned Power Grid Corporation of India as it focuses on forming the Northern, Eastern, Western, North Eastern and Southern Grids.

These regional grids were synchronously interconnected to the Central Grid on 31 December 2013 to enable transmission of surplus electricity between States in each region. This step was made to emphasize continuity in power supply throughout the nation but lacked the ability to remotely monitor the power supply.

\section{SYSTEM MODEL}

The idea presented here is focussed on monitoring continuous power supply, eliminating the inconsistencies and enhancing the reliability of the centralized systems to overcome the intermittent power cuts with the help of smart grids[3] and a dedicated workstation. Though the power shutdowns dismiss the mishaps at times, events ensued by it have a greater impact. One of the alternatives to minimize the impact is to make use of secondary resources which is efficient as well as lucrative in economic perspectives [4].

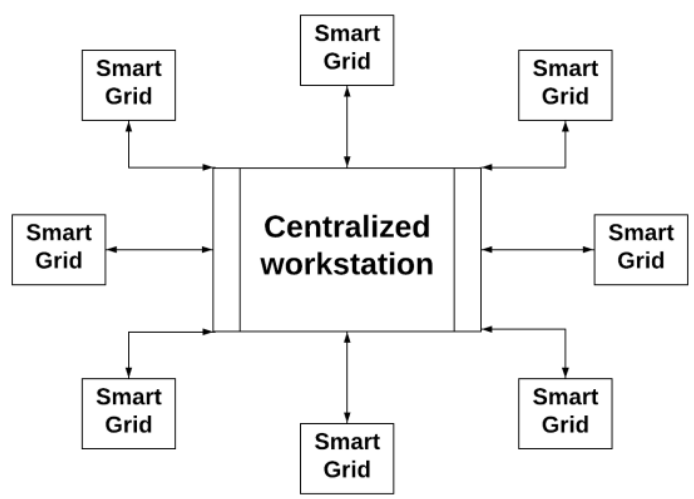

Fig 1: A centralized workstation

The main task in making use of secondary sources is to automatically switch from the primary source to the alternative sources of power in the absence of power in the primary line and infer if any faults or frailties have occurred. This is followed by summoning the electricity boards to examine into the oversights, errors and inaccuracies that would have befallen. Switching responsibility is born by the automatic phase selector. The sole of sending intimations regarding the power status is vested in the smart grid. The centralized workstation receives the data sent over the network by the smart grids via the GSM module and implies the necessary actions to be taken further with its advanced and efficacious algorithms.

\section{PROBLEM STATEMENT}

Outages are often due to aging and poor maintenance of infrastructure and deficient monitoring of power supply. Poor reliability of grids and dearth of uninterrupted and continuous power supply happen to be the main causes. Inefficacious monitoring puts the hap of efficient utilization of alternative sources into vain.

\section{SOLUTION}

Improved monitoring is an elementary step. Consolidated workstations add up to achieve the surveillance of continuous power supply. The tried and true grids shall be obtained by the implementation of smart grids. These smart grids ensure a reliable and cost-effective power supply enabled by robust, self-healing systems. It also imparts the integration of renewable energy resources to enhance energy independence hence yield more sustainable power. Dynamic capabilities of the smart grid allow greater information gathering and control which help an electricity system to spot problems and re-route them.

Development of Wireless Network smart grid, which monitors the current flow, is capable of sending data to the workstations continuously, where the algorithms predict and detect the problems in transmission lines based on the received data. This would include the possibilities of power failure, the approximate location of the mishap, etc. Further helping in eliminating the defects initially or to isolate the flaws from the lines completely.

\section{ANALYSIS}

The device includes Automatic phase selector, Raspberry Pi, Step Down Transformers and GSM module. The automatic phase selector makes sure that there is no interruption in the power supply while the Raspberry Pi monitors the flow in real time and sends the updates to the workstation using the GSM module; the workstation detects the faults with its advanced and complex algorithms. Once the fault is predicted, the workstation generates a warning token to the designated authorities based on the level of attention required.

The smart grids (Fig 2) are installed in the power substations from where the power is being supplied for domestic usage and are configured to transmit data continuously to the designated workstation. The workstation is engineered along with a user-friendly and highly interactive UI designed in such a way that the crew monitoring it can easily notice the changes happening in the transmission lines. Even though the fault detection is completely automated, the monitoring crew will be able to manually log the fault in case the fault goes undetected or wrongly detected [8].

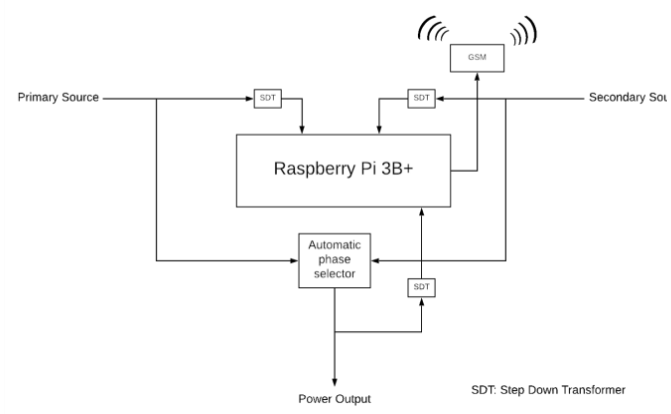

Fig 2: Smart grid 


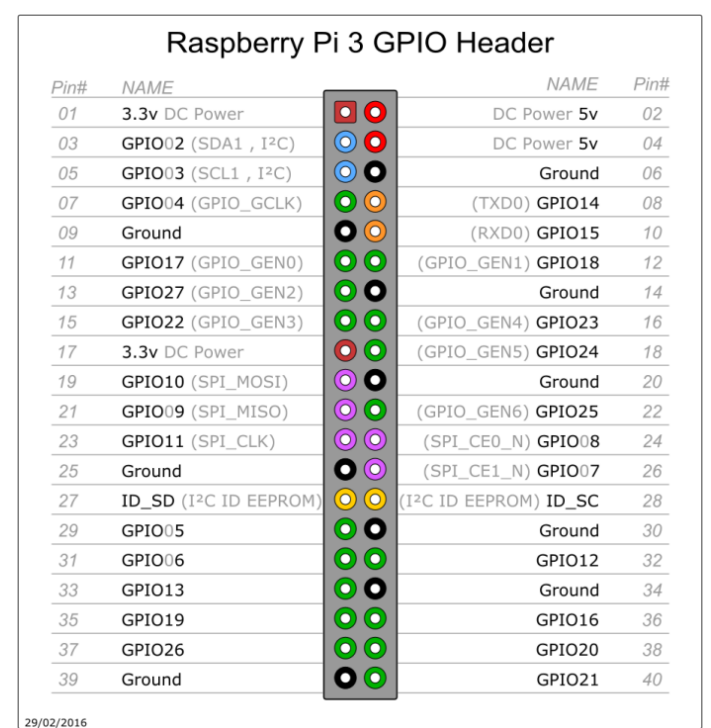

Fig 3: Raspberry Pi 3B+ pin diagram

The current detection in the smart grid is done using the GPIO (General Purpose Input Output) pins on the Raspberry $\mathrm{Pi} 3 \mathrm{~B}+$ (Fig 3). The voltage is stepped down to $5 \mathrm{~V}$ before it is connected to the system as the Raspberry $\mathrm{Pi}$ can only handle up to $5 \mathrm{~V}$. Even though it is being difficult to manage to detect faults like phase differences in current, continuous research is being done in order to ensure that all the known flaws can be avoided. Moreover, the Raspberry $\mathrm{Pi}$ is programmed to include its geo-location along with the transmitted data in order to make it easier to locate the fault when it is detected by the workstation [5], [6].

\section{EXPERIMENTATION}

The workstation being highly configured makes sure that it receives the information from all the smart grids with less latency and minimal or zero data loss. WN can not only efficiently prevent and lessen power equipment accidents when combining with the conductor temperature, environmental and meteorological real-time online monitoring, but can also provide data to support transmission efficiency improving and increasing the dynamic ability for broadcast lines [7].

\section{CONCLUSION}

The present power scenario in the country is a huge hassle that needs to be overcome. With reference to the current power, the demand is on the higher end when related to supply, making the nation's economic growth lag behind. Smarter electricity helps managing demand on a large scale. This smart grid is being developed with the most advanced and latest platforms using the latest technologies. It is designed in such a way that it is totally customizable and can adapt future technologies and is flexible for upcoming platforms. Raspberry Pies are being configured and programmed to monitor the power supply. Automatic phase selectors are dealt in switching to the alternate source when the power in the primary source is not available, thereby secondary sources are utilized efficiently. Smart grids are a better choice in the integration of alternative power sources as well. Thus the outages and the blackouts are considerably reduced. [8].

\section{FUTURE ENHANCEMENTS}

In the near future, the device will be programmed to predict faults in the power supply with more accuracy. Heavy rains and varied climatic conditions are also the reasons behind the failure of power systems. In order to obviate such problems, the proposed system can be further enhanced by integrating various sensors like rain sensors and barometers. Moreover, the system can be programmed to fetch details from weather reports and with the combination of all these inputs, the power systems can be put into a safe mode of operation which is designed to avoid all the adversities that can occur in these circumstances, simultaneously focussing more on the maintenance of the smart grids [7], [9].

\section{ACKNOWLEDGEMENT}

We thank, Karnataka Science and Technology Promotion Society(KSTePS) for giving us the opportunity to work on the project "WSN Based smart grid for Continuous power supply " submitted under Vision Group of Science and Technology (VGST ) scheme of KFIST level (1) for the financial year 2016- 2017.

\section{REFERENCES}

1. Suresha, Parvathi C, "WSN Based Continuous Power Supply For Agriculture Using Smart Grid",International Journal of Mechanical and Production Engineering Research and Development (IJMPERD), ISSN (P): 2249-6890; ISSN (E): 2249-8001, Vol. 8, Special Issue 2, Nov 2018, 295-302 (C) TJPRC Pvt. Ltd.

2. https://en.wikipedia.org/wiki/National_Grid_(India)

3. https://en.wikipedia.org/wiki/Smart_grid

4. Md. Moyeed Abrar, "Power cut off and Power Blackout in India a Major threat- An Overview", International Journal of Advancements in Research \& Technology, Volume 5, Issue 7, July-2016 8 ISSN 2278-7763

5. https://www.raspberrypi.org/forums/viewtopic.php?t=112813

6. https://www.elprocus.com/gsm-architecture-features-working/

7. https://bescom.org/en/power-outage/

8. https://www.w3schools.in/python-tutorial/gui-programming/

9. https://www.voanews.com/a/india-blackout-highlights-powerproblems-in-developing-countries/1452613.html

\section{AUTHORS PROFILE}

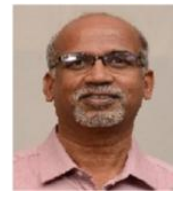

Dr. Suresha is currently working as Principal in Sri Venkateshwara College of Engineering, $\mathrm{He}$ is an experienced educator with a demonstrated history of working in the higher education industry. His research interests are Distributed Sensor Networks, Wireless Sensor Networks and Network Security. He introduced meditation classes to engineering students at Sri Venkateswara College of Engineering, Bangalore (SVCE). He has received Rs. Twenty lakh funding from VGST-Vision Group of Science \& Technology, Karnataka under K-FIST level-1 for infrastructure development on WSN Based smart grid for Continuous power supply of 2016-17. He has received Principal of the year award in 2018.

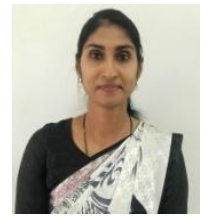

Parvathi C is working as an Asst. Professor, Department of Computer Science and Engineering, Sri Venkateshwara College of Engineering, Bangalore. She has pursued MTech (PhD). She has an experience of 12 years in teaching and 2 years as Human resource executive. interested in educating rural students. She has published 6 papers in International journals and 2 papers in International Conference, secured Silver Certificate Award from Infosys, Bengaluru, 2014 and Bronze Certificate in 2015 in Contest GURU Event. She has secured best teacher award from the Institute of Scholars for the year 2018 . 


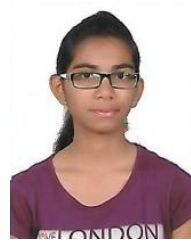

Monitoring.

Ashritha C is currently pursuing B.E. in Computer Science and Engineering from the Department of Computer Science and Engineering, Sri Venkateshwara College of Engineering, Bangalore. Her area of interest are Radars, Micro Processors, Programming and Robotics. She has successfully completed development of 2 client projects and working on Wireless Network Smart Grids for Continuous Power Supply and

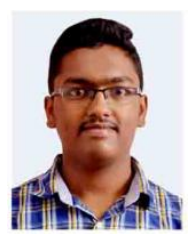

Abhay R Muni is currently pursuing B.E. in Computer Science and Engineering from the Department of Computer Science and Engineering, Sri Venkateshwara College of Engineering, Bangalore. His area of interest are Networks, Cyber Security, Python Programming, Shell Scripting, Artificial Intelligence and Machine Learning.. He has successfully completed development of 2 client projects and working on Wireless Network Smart Grids for Continuous Power Supply and Monitoring.

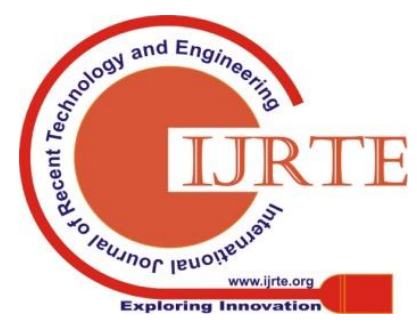

